\title{
AC 2007-136: TO ENCOURAGE AND EXCITE THE NEXT GENERATION OF ENGINEERS THROUGH HUMAN-ROBOT INTERACTION PROJECTS FOR SPACE EXPLORATION
}

\section{Ayanna Howard, Georgia Institute of Technology}

Dr. Ayanna Howard has a strong commitment to advancing research centered around transplanting human "smarts" into robotic systems. Her research has resulted in over 60 written works in a number of projects - from landing on Mars to autonomous rover navigation. To date, her unique accomplishments have been documented in over 12 featured articles - including being named as one of the world's top young innovators of 2003 by the prestigious MIT Technology Review journal and in TIME magazine's "Rise of the Machines" article in 2004. From 1993-2005, Dr. Howard was at NASA's Jet Propulsion Laboratory where she led research efforts on various robotic projects. Following this, she joined the School of Electrical and Computer Engineering at Georgia Tech in 2005 and founded the Human-Automation Systems (HumAnS) Laboratory. In addition, she is actively involved in inspiring the next generation of engineers and scientists through active mentoring and training.

\section{Eva Graham, NASA's Jet Propulsion Laboratory}

Eva Graham is the current manager of NASA/JPL's Minority Education Initiatives, in which she works to make sure that groups typically underrepresented in science and engineering will have a chance at success. She began working at JPL right after college, setting up organic chemistry reactions, then again after obtaining her master's degree from Tuskegee University in Alabama, in JPL's analytical chemistry lab. After teaching high school biology and chemistry for three years and doing outreach for undergraduate scholarships with the National Action Council for Minorities in Engineering, she found herself back at JPL in 2001. Her department, which is one arm of NASA/JPL's education branch, focuses on supporting historically African-American colleges, tribal colleges and those that serve large Latino populations. 


\title{
To Encourage and Excite the Next Generation of Engineers through Human-Robot Interaction Projects for Space Exploration
}

\begin{abstract}
The robotics field represents the integration of multiple facets of engineering and science - from mechanical construction to intelligence programming to science data analysis. It is an ideal opportunity to showcase the relationship math and science have on tangible real-world applications. Unfortunately, the multi-disciplinary nature of robotics creates difficulty in using robotics as a pedagogical tool for the diverse learning styles present in different disciplines. The infusion of low-cost robotic platforms has alleviated some of these difficulties, but without an experienced and knowledgeable robotics instructor, the full benefit of this infrastructure cannot always be exploited. Yet, the field of robotics provides an ideal educational platform to encourage and excite undergraduate students. To capitalize on these benefits, we have developed human-robot interaction modules that combine human-robot interaction technology and space exploration problem sets into an intense design experience, in which emphasis is placed on the non-traditional skills needed for addressing challenging technical problems of the $21^{\text {st }}$ century. In this paper, we discuss our intense "boot-camp" learning process that uses these modules for training diverse populations of undergraduate students. The underlying model is that by providing undergraduate students the ability to understand the linkage between theory and real-world applications, it increases their motivation to remain in a STEM-related major.

Discussion on the approach is presented in this paper and validated through implementation with a pilot student population to provide supportive evidence of the observed benefits.
\end{abstract}

\section{Introduction}

An essential element of NASA's education mission to inspire the next generation of explorers, requires that the public is engaged through the implementation of innovative approaches such as informal and formal education methods, science outreach, and research development efforts. ${ }^{1}$ One of the direct outcomes of this focus is to contribute to the nation's educational goals by creating a bridge to provide students access to the research and development activities involved in space exploration, as well as to support and develop a pipeline program to encourage the next generation of engineers and scientists. Of special interest is to ensure that robotics research knowledge derived from exploration missions and programs is transferred to the educational arena. $^{2}$

The current exploration vision of NASA is to develop the technology and infrastructure required to send humans back to the Moon and Mars. ${ }^{3}$ Some of the technologies that must be matured are those that are needed to support combined robot/human crews for establishing a base on the Moon in a safe and cost effective manner. Addressing challenging engineering problems such as this provide an opportunity to demonstrate real-world applications for science and technology outside of the classroom environment. Thus, the primary goal as discussed in this paper is to use robotics and space exploration problem sets as a basis for an intense design experience, in which students begin to develop and understand the relevant skill sets that are needed for tackling 
challenging problems of the $21^{\text {st }}$ century. In essence, we can use the non-traditional skill sets learned in robotics research, especially those of collaboration and applied experimentation, and NASA's ability "to inspire the next generation of explorers" 3 in order to encourage, excite, and retain students in the science, technology, engineering, and math (STEM) fields.

In this paper, we will discuss details on an intense "boot-camp" learning process that combines human-robot interaction technology and space exploration applications within a design workshop setting. Section 2 provides background information for developing the design experience, and addresses the reasons why infrastructure, such as low-cost robotic platforms, is essential for success. Section 3 discusses the pedagogical process for our approach. A description of the human-robot interaction modules for the design workshop is documented in Section 4. Finally, results from pilot student populations and concluding remarks are provided in Sections 5 and 6.

\section{Background}

The traditional use of robotics in the undergraduate curriculum focus on three main components: concept learning, laboratory exercises, and projects/competitions. ${ }^{4}$ For concept learning, the primary limitation resides in the fact that students must undergo learning theories that are not necessarily compatible with their current knowledgebase. In other words, computer science majors are required to understand the concept of gear ratios before delving into the theory of behavior-based control, cognitive science majors are introduced to engineering concepts before they can practice designing complex neural networks, and mechanical engineers are required to study the concepts of microprocessors before investigating aspects of structure and design.

Laboratory exercises provide hands-on activities that enforce the learning concepts by coupling them with a robotics application. ${ }^{5}$ Traditional robotics courses use the laboratory exercises to build a robotic system or application from a beginning foundation and are an effective means for linking learned knowledge to real-world application. They suffer though from two main challenges. The first challenge involves determining how to structure the exercises to both reinforce the learned knowledge while ensuring that it still excites and motivates the student. This involves determining what trade-offs need to be made between designing open-ended problems that may overstress the student versus step-by-step (routine) problems that may ultimately not stimulate their intellectual ability. The second challenge involves determining how to support the cross-disciplinary curriculum that is required to implement most robotic laboratory exercises, while still developing the complex skill set relevant to the individual student. As a means of addressing these two challenges, the use of low-cost robotic platforms allows students to work with pre-designed robots that have sufficient complexity and capability to engage them at any learning level necessary. Low-cost robotic platforms thus allow a multi-disciplinary approach for robotics education to successfully address the diverse learning styles present in undergraduate education.

Robotics competitions, the third component of robotics in the undergraduate curriculum, provides a forum for students to design and build robots to solve a specific engineering problem. ${ }^{6}$ Programs such as the AAAI Robot Competition and RoboCup are efforts that are effective in exciting students about engineering and science. Small contests, held at the local university level, provide a means to guide students into developing a final product (sometimes in a class or laboratory environment). However, these contests typically require students to be technically 
advanced or supported by highly-technical mentors or graduate students. In addition, they tend to involve a large amount of physical and mental effort in order for the students to develop enough relevant skills to bridge the gap to mastery of the competition goals.

\section{Motivation and Approach}

In this work, our primary goal is to provide a mechanism that can 1) encourage and excite the next generation of engineers and 2) train students to understand the underlying skill sets associated with the problem-solving process, especially those highlighted by teamwork, system engineering, and hands-on experimentation. Our premise is that by providing undergraduate students the ability to understand the linkage between theory and real-world applications, it increases their motivation to remain in a STEM-related major. And by training students on the non-traditional skill sets required in robotics, it improves their ability to problem-solve. Our approach therefore combines human-robot interaction technology and space exploration problem sets into an intense design experience, in which emphasis is placed on the non-traditional skills needed for addressing challenging technical problems of the $21^{\text {st }}$ century.

One of the key components to robotics research is learning to apply abstract concepts to realworld mechanical systems that operate in a dynamic world environment. Just as it is generally not feasible nor functional to design a generalized algorithm that can solve all problems potentially encountered by a robot, using robotics as a one-stop pedagogical tool does not work to adequately tackle the different learning styles and different technical discipline areas found in undergraduate education. Two fundamental factors differentiate each student's learning style, namely their intrinsic skill set and their externally learned knowledgebase. A student's intrinsic skill set is typically influenced by their designated discipline or major, whether cognitive science, electrical engineering, mechanical engineering, or other. The student's knowledgebase is normally constructed through experience, i.e. the years of both formal and informal instruction pursued by the student. The approach that we adopt for training students addresses the diversity in undergraduate learning styles by differentiating between these two factors. Our approach involves the implementation of three main sequences: framing, presentation, and modeling. Framing focuses on understanding the target student audience and identifying each students' respective learning style. Presentation involves categorizing and selecting classes of robotic problems relevant to the learning styles of the target audience. Modeling focuses on using examples to move the novice towards mastery of the subject matter while still motivating the more experienced students.

\section{A. Framing Sequence}

Framing is a popular context used in social sciences that recognize that individuals involved in a complex problem will define it in different ways. ${ }^{7}$ As an example, a computer science major may view robotics as an interesting computing application or as a complex hardware platform that never responds exactly as desired to software commands. On the other hand, a mechanical engineering major may see robotics as a structural design problem or a terrifying journey into the programming world. Framing involves identifying the mindset of the student learner. The most efficient manner to determine an individuals framing is to ask direct questions. In an effort to gain an overall understanding of how much students actually understand about robotics, surveys 
are given asking how much exposure students have had with the overall subject matter (Figure 1).

The influence of framing in the problem-solving domain is highlighted by the fact that the problem solving process involves analyzing a problem in different ways. Group problemsolving, a derived benefit of the framing sequence, is a process that can be used to enhance the diversity of thought necessary for viewing a problem in different ways, subsequently producing revolutionary solutions. In fact, group problem-solving has been shown be an effective means to develop new mission concepts and technologies for some of the NASA centers. The Jet Propulsion Laboratory uses the Advanced Projects Design Team (APDT) ${ }^{8}$ construct that brings together mission designers and engineers with diverse expertise to assess the potential of new technologies and perform rapid and thorough designs and reviews of varying space missions. And the Integrated Mission Design Center (IMDC) ${ }^{9}$ was developed at NASA-Goddard Space Flight Center to allow mission designers and engineers to develop advanced space mission design concepts to increase scientific value for NASA and its customers.

Based on this framing construct, the focus of the boot-camp design experience capitalizes on a collaborative learning process, which involves teaming students together who have different prior knowledge-bases and skill sets and providing group-based assignments. The students with the greatest amount of prior knowledge are teamed up with those who have very limited robotics experience and groups are constructed to consist of students having diverse skill sets, which is categories by their respective disciplines. This type of grouping is based on Vygotsky's theory that knowledge should be co-constructed between two or more people in order to support the acquisition of new knowledge. ${ }^{10}$ This limits the potential for cognitive load and maintains individual and group motivation by "using technology to problem solve ... and to promote collaboration and cooperation among learners". 11

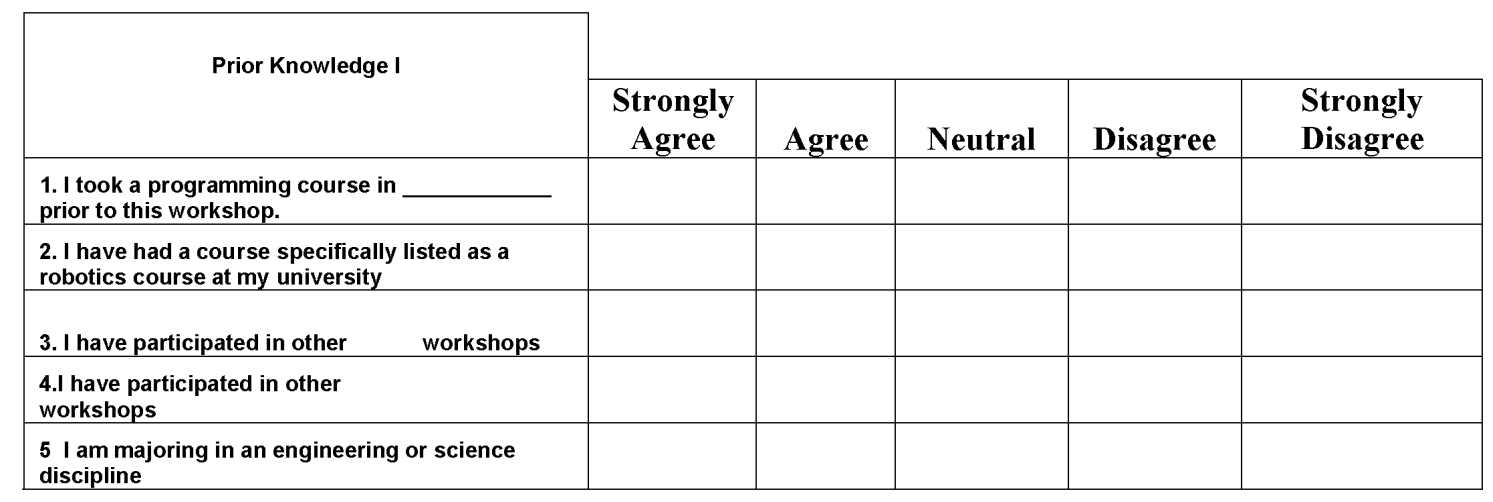

Figure 1. Pre-survey to determine an individual's framing

\section{B. Presentation Sequence}

The presentation sequence involves the implementation of three major phases - 1) presentation of concepts, 2) example sharing and worked examples, and 3) practice and feedback.

The first phase, presentation of concepts, involves providing sufficient background information on the design experience. Since our focus is to have students solve space exploration problems 
using human-robot interaction (HRI) technology, we first provide a brief lesson covering the underlying concepts behind both Space and HRI (Figure 2). The lessons are designed to provide sufficient background to understand the problem, without getting overloaded with the theory that forms the basis of the problem.
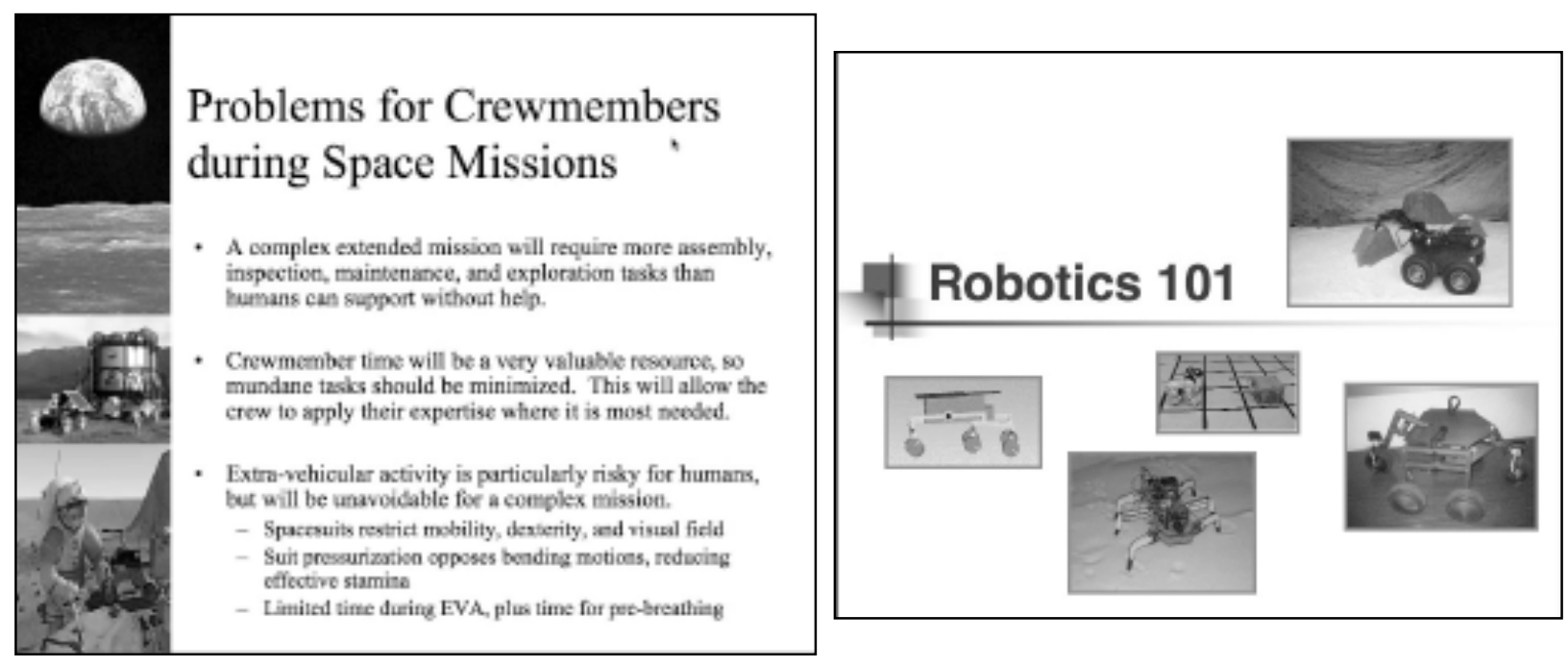

Figure 2. Sample of course handouts for presenting design concepts

The second phase, example sharing and worked examples, involves the instructors linking the presented concepts to example problems that are relevant to the course focus. This starts the process of training them to attend to the appropriate information as they begin to recognize problems that they may need to solve. The discussion of appropriate problems and the given definitions will again allow the students to use storage techniques and build schema for appropriate problems as they move toward solution orientation. ${ }^{13}$ Group brainstorming has been shown to be very useful when used to engage the experience of all team members during problem solving activities. ${ }^{12}$ As such, a group brainstorming session that involves all participants is implemented for the example sharing and worked examples phase (Figure 3).

The last phase, practice and feedback, involves the students becoming involved with a problem associated with the specific content area and evaluating their understanding of why the presented problem is appropriate. This process promotes the theory of meaningful learning, the process in which a student makes sense of new information such that it is possible to effectively store it in long term memory through positive feedback. ${ }^{13}$ In this phase, the individual teams develop concepts for addressing any of the problems identified during the group brainstorming session (Figure 4). At this point, the teams are encouraged to communicate their ideas and ask questions of the instructors in order to get feedback on the team's progress toward developing a solution. 


\section{Group Brainstorming}

Project Description: NASA has decided to establish a moon base as the precursor step to sending humans to Mars. This must be accomplished in a safe and cost effective manner. The strategy is to reduce cost and danger by $\mathrm{f}$ ist sending in robots to prepare the way, then following them with humans to establish a combined humans-robots team.

Scenario: The year is 2020. A colony of robots is operating on the moon. The $\mathrm{f}$ ist astronauts have just landed. The HumAnS user interface, operational by either mission engineers on Earth or the astronaut, facilitates command and control of the robot colony.

Objectives: Research to enable the robots to work with the astronauts has not been developed. Available infrastructure is not suff cient and errorprone. There are currently many problems and yet it is crucial that astronauts and robots can work together with existing technology to build a selfsuff cient moon base.

Tasks: Your team is tasked with developing a technology that can enable construction of the moon base. You don't have to solve the entire problem, just a technology module related to the larger objective. Be prepared to defend your case and sell it to NASA decision makers.

\section{Group Brainstorming}

What tasks/steps are required for constructing a base on the Moon?

Which tasks do you think are better suited for a robot to perform and why?

Which tasks do you think are better suited for a human to perform and why?

What problems can you think of in having the robot perform these tasks?

What problems can you think of in having the human perform these tasks?

What are some ways of addressing the robot problems?

What are some ways of addressing the human problems?

Figure 3. Visuals associated with group brainstorming phase

\section{Team Brainstorming}

- Choose one of the tasks/steps required in constructing a Moonbase

- Choose one of the problems that hinder completion of this task with either human or robot

- Determine how the problem can be solved using both humans and robots together

- What tasks/steps are required for solving the problem using humans and robots?

- Choose one of these problem tasks/steps

- Determine how you can solve it using your available resources (AIBO robot, HumAnS interface, etc.)

Figure 4. Visual associated with team brainstorming session

\section{Modeling Sequence}

The Modeling Sequence consists of three primary phases. Once the framing and presentation sequences are complete, the students must be given the ability to model their own understanding of the learned knowledge. This first phase, sharing a worked example while modeling the technique, involves implementing the solution identified during the team brainstorming session. This will allow students to build on the previous information given to increase their level of expertise required to design a solution to a problem. Following this phase is practice with 
feedback in which the instructor randomly observes each team as they work and offers positive feedback and guidance. The third phase, group feedback, requires that each team present their work to the group.

Using this approach consisting of Framing, Presentation, and Modeling, we now present results of its implementation with a pilot student population.

\section{Human-Robot Interaction Modules}

Advances in space science and technology have enabled humanity to reach a stage where we are able to send manned and unmanned vehicles to explore nearby planets. However, it is not always easy or feasible to expect all mission related tasks to be accomplished by astronauts alone. The presence of robots that specialize in different tasks would greatly enhance the mission capabilities and enable better overall performance.

Although determining the kinds of robots necessary for the mission is important, ensuring successful communication, feedback and the possibility of remote interaction between the human and robot agents are equally important. The approach we take is to enable human-robot collaboration at a very high-level of interaction, rather than building a simple remote control mechanism. In other words, we hope to make the human-robot interaction as close as possible to a human-human interaction.

The purpose of the human-robot interface is primarily designed for operations in space, where resources may be scarce. The software is built such that a physical robot connected to a virtual robot controllable through the interface can autonomously perform a number of basic primitives (such as path planning, unsupervised traversal, etc.), but also allow the human to be flexible in the type of interaction - from direct tele-operation of the robotic vehicle to a collaborative role in which the human communicates to the robot through a natural language processor.

As part of the design experience, each team is provided 1-2 robots, basic robot operating instructions, and time for each member of the team to individually explore the interface. Each team is then tasked with realizing their solution to the problem identified through the teambrainstorming session using the human-robot interface module and associated robot platform (Figure 5). 


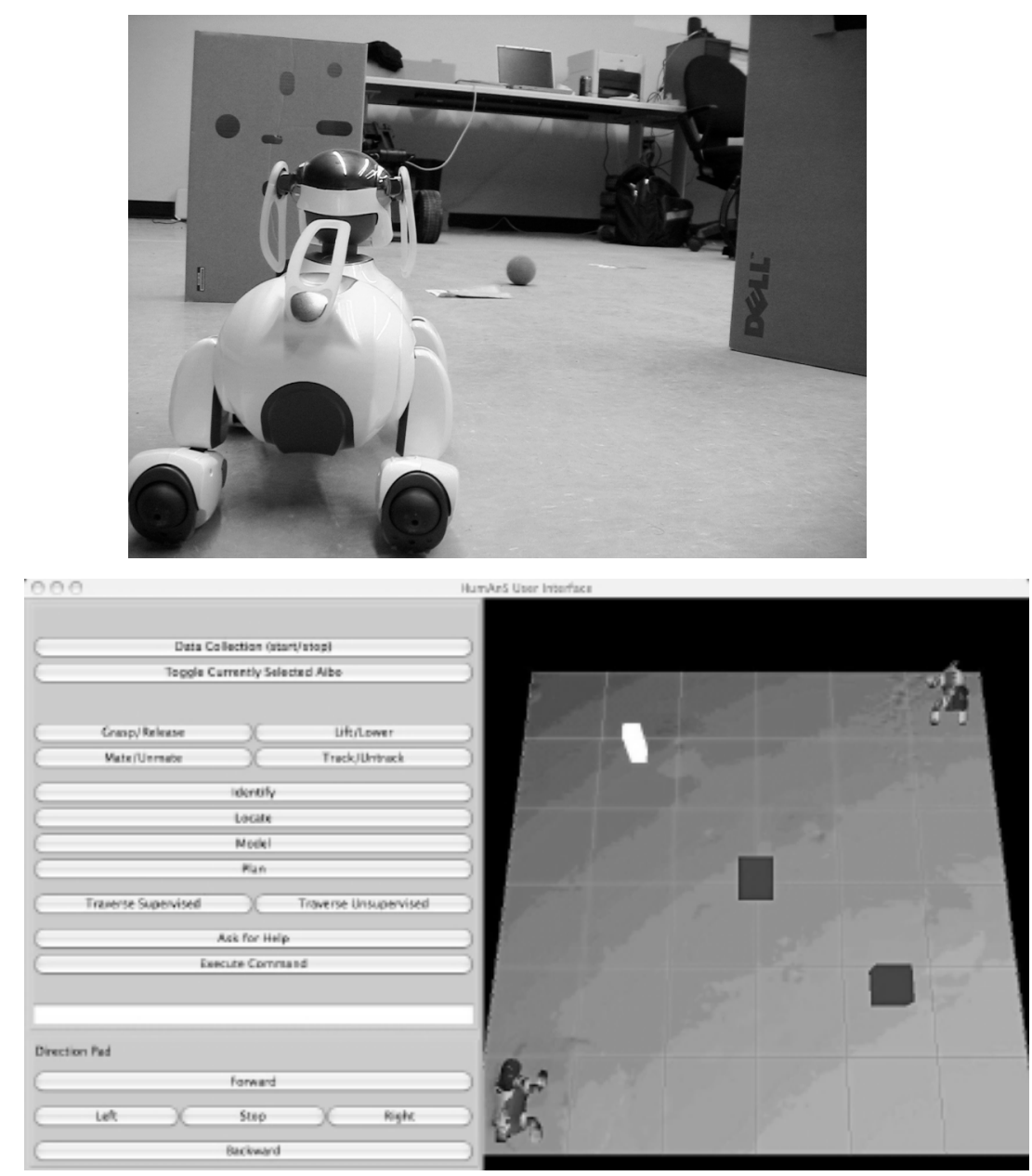

Figure 5. Human-Robot Interaction Modules

\section{Results from pilot student populations}

To evaluate the benefits of the design workshop, 30 students, over a 3-week period participated in one of three workshop sessions. Each team consisted of 3-4 students with varied disciplines, class levels, gender, and culture. The problems selected by the students ranged from transportation of material on the lunar surface to clearing of a lunar site for habitat construction. At the conclusion of the workshop, a survey was provided as shown in Figure 6 . We compile metrics on the three most significant questions:

- Question 1: I feel more encouraged to pursue robotics - either in my senior design project, in undergraduate research, or in graduate school.

- Question 2: The workshop activities helped me to understand the real world applications of engineering and science.

- Question 3: The workshop activities changed my belief in what is required to succeed in engineering or science. 
The associated mean response was 3.9 with a standard deviation of 1.1 (Question 1), 4.6 with a standard deviation of 0.5 (Question 2), and 3.3 with a standard deviation of 0.9 (Question 3).

Thus, the students strongly agree that the workshop session provides a good understanding of the real world applications of science and technology. They agree that they are encouraged to pursue robotics later in their educational endeavors, but only slightly agree that the workshop changed their beliefs in what is required to succeed in engineering or science.

\begin{tabular}{|c|c|c|c|c|c|}
\hline \multirow[t]{2}{*}{$\begin{array}{l}\text { Part II. Academic Affect } \\
\text { Note: we are interested in understanding how } \\
\text { being selected and participating in this workshop } \\
\text { might impact you academically. }\end{array}$} & \multirow[b]{2}{*}{$\begin{array}{l}\text { Strongly } \\
\text { Agree }\end{array}$} & \multirow[b]{2}{*}{ Agree } & \multirow[b]{2}{*}{ Neutral } & \multirow[b]{2}{*}{ Disagree } & \multirow[b]{2}{*}{$\begin{array}{l}\text { Strongly } \\
\text { Disagree }\end{array}$} \\
\hline & & & & & \\
\hline \multicolumn{6}{|l|}{$\begin{array}{l}\text { 4. I believe that I can use what I've learned in this } \\
\text { workshop in my up coming semester. }\end{array}$} \\
\hline \multicolumn{6}{|l|}{$\begin{array}{l}\text { 5. I plan to share what l've learned with } \\
\text { classmates and/or my regular study group. }\end{array}$} \\
\hline \multicolumn{6}{|l|}{$\begin{array}{l}\text { 6. I feel more encouraged to continue in my } \\
\text { engineering and science discipline. }\end{array}$} \\
\hline \multicolumn{6}{|l|}{$\begin{array}{l}7 . \text { I met new people during the workshop that I will } \\
\text { keep in contact with. }\end{array}$} \\
\hline \multicolumn{6}{|l|}{$\begin{array}{l}\text { 8. I feel more encouraged to pursue robotics - } \\
\text { either in my senior design project, in } \\
\text { undergraduate research, or in graduate school. }\end{array}$} \\
\hline \multicolumn{6}{|l|}{$\begin{array}{l}\text { 9. I am not currently an Engineering or Science } \\
\text { major but plan to change my major or transfer to } \\
\text { another university because of what I learned in } \\
\text { this workshop. }\end{array}$} \\
\hline \multicolumn{6}{|l|}{$\begin{array}{l}\text { 10. The workshop activities helped me to } \\
\text { understand the real world applications of } \\
\text { engineering and science. }\end{array}$} \\
\hline \multicolumn{6}{|l|}{$\begin{array}{l}\text { 11. The workshop activities changed my belief in } \\
\text { what is required to succeed in engineering or } \\
\text { science. }\end{array}$} \\
\hline $\begin{array}{l}\text { 12. The workshop activities showed me that I am } \\
\text { capable of doing research. }\end{array}$ & & & & & \\
\hline
\end{tabular}

Figure 6. Post-survey to determine affect of design workshop

\section{Conclusions}

In this paper, we present an approach that brings the hands-on excitement of space robotics projects into the university environment to encourage and retain undergraduate students. By encompassing the theories of framing, presentation, and modeling, we can address the differences in knowledge as well as learning modalities. The results have shown that through the design concept, we can achieve our primary goal of 1) encouraging and exciting the next generation of engineers and 2) training students to understand the underlying skill sets associated with the problem-solving process. Future work will involve structuring the design workshop to allow web-based dissemination and ease-of-use for middle and high school teachers. 


\section{References}

1. C. Bowman, Robotics Education Project Website, http;//robotics.nasa.gov., 2004.

2. A. Howard and E. Graham, "Bridging the Gap between Space Robotics Research and Robotics Education," AAAI Symp. on Accessible, Hands-on AI/Robotics Education, San Jose, CA., pgs. 126-128, 2004.

3. NASA's Vision, "Administrator unveils future NASA vision and renewed journey of learning," http://www1.msfc.nasa.gov/NEWSROOM/news/releases/2002/02-087.html

4. Z. Dodds, L. Greenwald, A. Howard, S. Tejada, J. Weinberg, "Components, Curriculum, and Community: Robots and Robotics in Undergraduate AI Education," AI Magazine, Vol. 27, pp. 11-22, Spring 2006.

5. D. Kumar and L. Meeden, "Robots in the Undergraduate Curriculum," Proceedings of the Third Annual Consortium for Computing in Small Colleges Northeastern Conference, Journal of Computing in Small Colleges, John G. Meinke (editor), Volume 13(5), 1998.

6. R. Murphy, "Using Robot Competitions to Promote Intellectual Development," AI Magazine, 21(1), pgs. 77-90, 2000 .

7. J. Roth and B.H. Sheppard, "Opening the black box of framing research: The relationship between frames, communication, and outcomes," Academy of Management Proceedings, 1995.

8. L. Sarsfield, The Cosmos on a Shoestring: Small Spacecraft for Space and Earth Science, RAND Document \#MR-864-OSTP, 1998.

9. Integrated Mission Design Center, Goddard Space Flight Center, http://imdc.nasa.gov/

10. D.H. Schunk, Learning Theories: An Educational Perspective (4th ed.). Upper Saddle River, NJ.: Pearson Merrill Prentice Hall, 2004.

11. S. Mills and R. Tincher, "Be the Technology: A Developmental Model for Evaluating Technology Integration," Journal of Research on Technology in Education. Eugene: Vol. 35, Iss; Pgs. 382- 397, 2003.

12. A.F. Osborn, Applied Imagination: Principles and procedures of creative thinking. Scribeners and Sons, New York, 1957.

13. M.H. Dembo and M. Eaton, "Self-Regulation of Academic Learning in Middle- Level Schools," The Elementary School Journal, Vol. 100, Iss. 5; Pgs. 473-475, 2000. 